PROCEEDINGS OF THE

AMERICAN MATHEMATICAL SOCIETY

Volume 128, Number 8 , Pages $2347-2355$

S 0002-9939(00)05241-2

Article electronically published on February 25, 2000

\title{
STATISTICAL EXTENSIONS OF SOME CLASSICAL TAUBERIAN THEOREMS
}

\author{
J. A. FRIDY AND M. K. KHAN
}

(Communicated by Albert Baernstein II)

\begin{abstract}
Hardy's well-known Tauberian theorem for Cesàro means says that if the sequence $x$ satisfies $\lim C x=L$ and $\Delta x_{k}=O(1 / k)$, then $\lim x=L$. In this paper it is shown that the hypothesis $\lim C x=L$ can be replaced by the weaker assumption of the statistical limit: st-lim $C x=L$, i.e., for every $\epsilon>0$, $\lim n^{-1}\left|\left\{k \leq n:\left|(C x)_{k}-L\right| \geq \epsilon\right\}\right|=0$. Similarly, the "one-sided" Tauberian theorem of Landau and Schmidt's Tauberian theorem for the Abel method are extended by replacing $\lim C x$ and $\lim A x$ with st-lim $C x$ and st-lim $A x$, respectively. The Hardy-Littlewood Tauberian theorem for Borel summability is also extended by replacing $\lim _{t}(B x)_{t}=L$, where $t$ is a continuous parameter, with $\lim _{n}(B x)_{n}=L$, and further replacing it by $\left(B^{*}\right)$-st-lim $B^{*} x=L$, where $B^{*}$ is the Borel matrix method.
\end{abstract}

\section{INTRODUCTION}

In 3] Fast defined statistical convergence of a complex number sequence $x$ (denoted st-lim $x=L$ ) by

$$
\lim _{n} \frac{1}{n}\left|\left\{k \leq n:\left|x_{k}-L\right| \geq \epsilon\right\}\right|=0
$$

for every $\epsilon>0$, where the vertical bars denote the cardinality of the set which they enclose. In [5] a Tauberian theorem was given for statistical convergence:

if st-lim $x=L$ and $\Delta x_{k}=x_{k}-x_{k-1}=O(1 / k)$, then $\lim x=L$.

NOTE: Throughout this paper $\Delta x_{k}$ will denote the "backward difference" $x_{k}-$ $x_{k-1}$, with $x_{-1}:=0$. The forward difference notation, which is frequently used in summability theory, is inconvenient when working with the one-sided Tauberian conditions.

Let $C x$ denote the Cesàro mean of order 1, i.e., $(C x)_{n}=(1 / n) \sum_{k=1}^{n} x_{k}$. In [6] Hardy presented one of the well-known Tauberian theorems for $C$ :

Theorem 1.1. If $\lim C x=L$ and $\Delta x_{k}=O(1 / k)$, then $\lim x=L$.

Since it is clear that $\lim x=L$ implies st-lim $x=L$, it follows that st-lim $C x=L$ is a weaker assumption than the hypothesis of Hardy's theorem. In Theorem 2.1 below we extend Hardy's theorem by using that weaker hypothesis.

Received by the editors March 5, 1998 and, in revised form, September 17, 1998.

1991 Mathematics Subject Classification. Primary 40E05.

Key words and phrases. Statistical convergence, Tauberian theorems.

(C)2000 American Mathematical Society 
In 11] Landau proved a stronger Tauberian theorem for $C$ by replacing $\Delta x_{k}=$ $O(1 / k)$ with the condition: $k \Delta x_{k} \geq-c$ for some $c>0$. We show that this onesided condition is also a Tauberian condition for statistical convergence, and use that result to extend Landau's theorem by again replacing $\lim C x=L$ with st-lim $C x=L$. Hardy and Littlewood's Tauberian theorem for Borel summability [8] states that if $x$ is Borel summable to $L$ and $\Delta x_{k}=O(1 / \sqrt{k})$, then $\lim x=L$. This theorem is extended similarly by replacing the hypothesis " $x$ is Borel summable to $L "$ with " $\left(B^{*}\right)$ st-lim $\left(B^{*} x\right)=L$ ", where $B^{*}$ denotes the Borel matrix method [13] (page 53), which includes $B$. A similar argument yields the statistical extension of the classical Tauberian theorem for the Euler-Knopp means [10.

\section{Tauberian theorems for Cesàro And Abel methods}

In order to extend Hardy's "big oh" theorem for Cesàro means, we first prove that the order estimate of the differences is preserved under the Cesàro transformation.

Lemma 2.1. If the sequence $x$ satisfies $\Delta x_{k}=O(1 / k)$, then $(\Delta C x)_{n}=O(1 / n)$.

Proof. For $n>1$ we have

$$
\begin{aligned}
n(\Delta C x)_{n} & =n\left\{\frac{1}{n} \sum_{k=1}^{n} x_{k}-\frac{1}{n-1} \sum_{k=1}^{n-1} x_{k}\right\} \\
& =\frac{1}{n-1}\left\{(n-1) \sum_{k=1}^{n} x_{k}-n \sum_{k=1}^{n-1} x_{k}\right\} \\
& =\frac{1}{n-1}\left\{(n-1) x_{n}-\sum_{k=1}^{n-1} x_{k}\right\} \\
& =\frac{1}{n-1} \sum_{k=1}^{n-1}\left(x_{n}-x_{k}\right) \\
& =\frac{1}{n-1} \sum_{k=1}^{n-1} \sum_{j=k+1}^{n} \Delta x_{j} \\
& =\frac{1}{n-1} \sum_{j=2}^{n}(j-1) \Delta x_{j} \\
& =\frac{1}{n-1} \sum_{j=2}^{n} O(1) \\
& =O(1) .
\end{aligned}
$$

Theorem 2.1. If the sequence $x$ satisfies st-lim $C x=L$ and $\Delta x_{k}=O(1 / k)$, then $\lim x=L$.

Proof. By Lemma 2.1. $(\Delta C x)_{n}=O(1 / n)$, which, by Theorem 3 of [5], is a Tauberian condition for statistical convergence. Therefore, st-lim $C x=L$ implies that $\lim C x=L$. Now Hardy's theorem [6] yields $\lim x=L$.

Our next theorem is an extension of Landau's theorem [11] that uses a onesided Tauberian condition. An argument similar to that just used for Theorem [2.1] 
will suffice, but it is necessary to prove first a one-sided Tauberian condition for statistical convergence.

Theorem 2.2. If the sequence $x$ satisfies st-lim $x=L$ and $k \Delta x_{k+1} \geq-c$, for some $c>0$ and for every $k$, then $\lim x=L$.

Proof. Assume st-lim $x=L$ and use Theorem 1 of [5] to get a sequence $y$ such that $\lim y=L$ and

$$
\lim _{n} \frac{1}{n}\left|\left\{k \leq n: y_{k} \neq x_{k}\right\}\right|=0 .
$$

Let $\{m(i)\}$ be the sequence of indices $k$ for which $x_{k} \neq y_{k}$, and assume that $m(i) \rightarrow \infty$. (Otherwise it is trivial that $\lim x_{k}=L$.)

We now assert that $\lim \sup x \leq L$. If not, there is a positive number $\epsilon$ such that $x_{m(i)} \geq y_{m(i)}+2 \epsilon$ for infinitely many $i$. For each such $i$ define $p(i)$ so that $x_{m(i)+p(i)}$ is the next term after $x_{m(i)}$ such that $x_{m(i)+p(i)}<y_{m(i)+p(i)}+\epsilon$. Thus it follows that

$$
x_{m(i)}-x_{m(i)+p(i)}>\frac{\epsilon}{2},
$$

for sufficiently large $i$. Since

$$
\frac{\left|\left\{k \leq m(i)+p(i): x_{k} \neq y_{k}\right\}\right|}{m(i)+p(i)} \geq \frac{p(i)}{m(i)+p(i)}
$$

the limit (1) implies that

$$
\lim _{i} \frac{p(i)}{m(i)}=0
$$

Using $k \Delta x_{k+1} \geq-c$, we write

$$
\begin{aligned}
x_{m(i)}-x_{m(i)+p(i)} & =\sum_{k=m(i)+1}^{m(i)+p(i)}-\Delta x_{k} \\
& \leq \sum_{k=m(i)+1}^{m(i)+p(i)} \frac{c}{k-1} \\
& \leq \frac{c p(i)}{m(i)} .
\end{aligned}
$$

Since (33) implies that the last quantity tends to zero as $i \rightarrow \infty$, for sufficiently large $i$ this contradicts (21), and we conclude that $\lim \sup x \leq L$.

To show that liminf $x \geq L$, suppose to the contrary that $x_{k} \leq y_{k}-\epsilon$ for infinitely many $k$. We also have $x_{i}=y_{i}$ for infinitely many $i$, and each such $i$ is followed by (a first) $m(i)$ where $x_{m(i)}<y_{m(i)}-\epsilon$. Therefore

$$
x_{i}-x_{m(i)}=y_{i}-x_{m(i)}>y_{i}-\left(y_{m(i)}-\epsilon\right)>\frac{\epsilon}{2}
$$

for $i$ sufficiently large. As in the above case, the fact that $-\Delta x_{k} \leq c /(k-1)$ implies that $x_{i}-x_{m(i)}$ tends to zero, which contradicts (4). Hence, we must have $x_{k}>y_{k}-\epsilon$ for $k$ sufficiently large, which implies that $\lim \inf x \geq \lim \inf y$, and the proof is complete.

With the theorem just proved we can now give an extension of Landau's Tauberian theorem [11] for $C$. 
Theorem 2.3. If $x$ is a sequence such that st-lim $C x=L$ and

$$
k \Delta x_{k+1} \geq-c, \text { for some } c>0 \text { and for every } k,
$$

then $\lim x=L$.

Proof. We first note that if $x$ satisfies (5), then $C x$ also satisfies (5); for, by the proof of Lemma 2.1,

$$
\begin{aligned}
n(\Delta C x)_{n} & =\frac{1}{n+1} \sum_{j=2}^{n+1}(j-1) \Delta x_{j} \\
& \geq \frac{1}{n+1} \sum_{j=2}^{n+1}(-c) \\
& =\frac{-n c}{n+1} \\
& \geq-c .
\end{aligned}
$$

Thus the hypotheses of Theorem 2.2 are satisfied by $C x$, so by Theorem $2.2 \mathrm{lim}$ $C x=L$. Now Landau's theorem [11] yields the conclusion that $\lim x=L$.

We now consider the Abel matrix methods $A_{\lambda}=\left[a_{n k}\right]$ defined by

$$
a_{n k}=\frac{1}{\lambda(n)}\left(1-\frac{1}{\lambda(n)}\right)^{k}, \quad k=0,1,2, \cdots,
$$

where $\lambda(n)$ is a strictly increasing sequence of real numbers so that $\lambda(0) \geq 1$. It is natural to suspect that under some conditions on the sequence $\{\lambda(n)\}$, the Abel matrix methods should preserve the $O(1 / n)$ difference order condition. The following lemma shows a bit more. It will be convenient for us to extend $\lambda$ to be defined over the interval $[1, \infty)$ by making it linear and continuous over $[j, j+1]$ for $j=1,2, \cdots$.

Lemma 2.2. Let $\lambda(n)$ be a strictly increasing sequence of real numbers tending to infinity such that

$$
\lim _{\delta \searrow 0} \lim _{n} \frac{\lambda(n+\delta n)}{\lambda(n)}=1 .
$$

If the sequence $x$ is in the domain of the Abel matrix method $A_{\lambda}$ and is of slow decreasing type, i.e.,

$$
\lim _{\delta \backslash 0} \liminf _{n} \min _{n \leq m<n(1+\delta)}\left(x_{m}-x_{n}\right) \geq 0,
$$

then the transformed sequence $z_{n}:=\left(A_{\lambda} x\right)_{n}$ is also of slow decreasing type.

Proof. In [1] Armitage and Maddox showed that if we let $y_{k}(p)=p^{k} / k$, then

$$
\begin{aligned}
(1-p) \sum_{k=0}^{\infty} x_{k} p^{k} & =\sum_{k=1}^{\infty}\left(x_{k}-x_{k-1}\right) p^{k} \\
& =a_{0}+\sum_{k=1}^{\infty} t_{k}\left(y_{k}(p)-y_{k+1}(p)\right), \quad 0<p<1,
\end{aligned}
$$


where

$$
t_{k}=\sum_{j=1}^{k} j\left(x_{j}-x_{j-1}\right)=\sum_{j=0}^{k}\left(x_{k}-x_{j}\right) .
$$

Armitage and Maddox further showed that if $x_{k}$ obeys the slowly decreasing Tauberian condition given above, then $t_{k}$ is bounded below by $-H k$ for some positive number $H$. Let $p_{n}:=1-\frac{1}{\lambda(n)}$. If the Abel transform of $x$ is denoted by $A(p)=(A x)_{p}$, then, for any $m \in[n, n+\delta n)$ and for some positive constant $H$, we have

$$
\begin{aligned}
A\left(p_{m}\right)-A\left(p_{n}\right) & =\sum_{k=1}^{\infty} t_{k} \int_{p_{n}}^{p_{m}} t^{k-1}(1-t) d t \\
& \geq-H \sum_{k=1}^{\infty} k \int_{p_{n}}^{p_{m}} t^{k-1}(1-t) d t \\
& =-H \int_{p_{n}}^{p_{m}}(1-t)^{-1} d t \\
& \geq-H \log \left(\frac{\lambda(n+\delta n)}{\lambda(n)}\right) .
\end{aligned}
$$

Hence, we see that

$$
\begin{aligned}
& \lim _{\delta \searrow 0} \liminf _{n} \min _{n \leq m<n(1+\delta)}\left(\left(A_{\lambda} x\right)_{m}-\left(A_{\lambda} x\right)_{n}\right) \\
\geq & \lim _{\delta \searrow 0} \liminf _{n}\left(-H \log \left(\frac{\lambda(n+\delta n)}{\lambda(n)}\right)\right) \\
= & -H \lim _{\delta \searrow 0} \limsup _{n} \log \left(\frac{\lambda(n+\delta n)}{\lambda(n)}\right) \\
= & 0 .
\end{aligned}
$$

Consequently, we see that the sequence $z_{k}=\left(A_{\lambda} x\right)_{k}$ obeys the slow decrease condition.

Remark 2.1. The above proof can be modified easily to show that the one-sided and the two-sided "big oh" difference Tauberian conditions are also preserved by the Abel matrix methods when $\lambda(n)=n^{\alpha}$ for some $\alpha>0$.

Our next theorem extends the classical Tauberian theorems of Hardy and Littlewood [9], 12].

Theorem 2.4. Let $A^{*}$ be the Abel matrix method $A_{\lambda}$ for which $\lambda(n)=n^{\alpha}$, for some $\alpha>0$. If the sequence $x$ satisfies st-lim $A^{*} x=L$ and $k \Delta x_{k+1} \geq-c$ for some $c>0$ and for every $k$, then $\lim x=L$.

Proof. By Lemma 2.2 and the above remark, we see that $\left(A^{*} x\right)_{n}$ obeys the onesided Tauberian condition. By Theorem [2.2] we must have $\lim \left(A^{*} x\right)_{n}=L$. Now Theorem 5 of [1] implies that $x$ is Abel summable to $L$, which then gives that $\lim x=L$.

Remark 2.2. We should remark that for the sake of simplicity we stated and proved the above result for the one-sided Tauberian condition. The following result, which 
is analogous to Theorem 2.4, may be proved for the slow decrease condition by using the statistical Tauberian results provided in [4]. This then extends the classical Tauberian theorem of Schmidt [14].

Theorem 2.5. Let $\lambda(n)$ be a strictly increasing sequence of real numbers going to infinity such that

$$
\lim _{\delta \searrow 0} \lim _{n} \frac{\lambda(n+\delta n)}{\lambda(n)}=1 .
$$

If the sequence $x$ satisfies $\operatorname{st}-\lim A_{\lambda} x=L$ and $x$ satisfies the slow decrease condition, then $\lim x=L$.

\section{TAUberian theOREMS FOR THE Borel METHOD}

For our final theorem we present a statistical extension of Hardy and Littlewood's Tauberian theorem [8] for Borel summability. Recall that the sequence $x$ is Borel summable to $L$ provided that

$$
\lim _{t \rightarrow \infty} e^{-t} \sum_{k=0}^{\infty} \frac{x_{k} t^{k}}{k !}=L .
$$

In order to use the notion of density that is inherent in statistical convergence, we replace the sequence-to-function Borel method with the Borel matrix method. Let $B^{*}$ be the summability matrix whose $n k$-th term is given by

$$
b_{n k}=\frac{e^{-n} n^{k}}{k !}
$$

We first show that $B^{*}$ preserves the appropriate order in the differences.

Lemma 3.1. If the sequence $x$ satisfies $\Delta x_{k}=O\left(k^{-1 / 2}\right)$, then

$$
\left(\Delta B^{*} x\right)_{n}=O\left(n^{-1 / 2}\right)
$$

Proof. It is easy to see that

$$
\left|x_{k}\right|=O\left(k^{1 / 2}\right)=O(k),
$$

and therefore, $\sum\left|x_{k}\right| n^{k} / k$ ! converges for each $n$, whence $\left(B^{*} x\right)_{n}$ is defined. Next note that in $B^{*}$, the $(n+1)$-th row is equal to the Cauchy product of the $n$-th row with the "1-th" row: more precisely,

$$
\begin{aligned}
\sum_{j=0}^{k} b_{n, k-j} b_{1, j} & =\sum_{j=0}^{k} e^{-n} \frac{n^{k-j}}{(k-j) !} e^{-1} \frac{1^{j}}{j !} \\
& =\frac{e^{-(n+1)}}{k !} \sum_{j=0}^{k} \frac{n^{k-j} k !}{(k-j) ! j !} \\
& =\frac{e^{-(n+1)}(n+1)^{k}}{k !} \\
& =b_{n+1, k} .
\end{aligned}
$$


Since each row sum of $B^{*}$ equals one, we can write

$$
\begin{aligned}
\left|\left(B^{*} x\right)_{n+1}-\left(B^{*} x\right)_{n}\right| & =\left|\sum_{i=0}^{\infty} \sum_{j=0}^{i} b_{1, j} b_{n, i-j} x_{i}-\sum_{k=0}^{\infty} b_{n, k} x_{k}\right| \\
& =\left|\sum_{j=0}^{\infty} b_{1, j} \sum_{i=j}^{\infty} b_{n, i-j} x_{i}-\sum_{k=0}^{\infty} b_{n, k} x_{k}\right| \\
& =\left|\sum_{j=0}^{\infty} b_{1, j} \sum_{k=0}^{\infty} b_{n, k} x_{k+j}-\sum_{k=0}^{\infty} b_{n, k} x_{k}\right| \\
& =\left|\sum_{j=0}^{\infty} b_{1, j} \sum_{k=0}^{\infty} b_{n, k}\left(x_{k+j}-x_{k}\right)\right| \\
& =\left|\sum_{j=0}^{\infty} b_{1, j} \sum_{k=0}^{\infty} b_{n, k} \sum_{i=1}^{j} \Delta x_{k+i}\right| \\
& \leq \sum_{j=0}^{\infty} b_{1, j} \sum_{k=0}^{\infty} b_{n, k} \sum_{i=1}^{j} \frac{K}{\sqrt{k+i}} \\
& \leq K \sum_{j=0}^{\infty} j b_{1, j} \sum_{k=0}^{\infty} b_{n, k} \frac{1}{\sqrt{k+1}} .
\end{aligned}
$$

We now assert that the last (inner) sum is $O\left(n^{-1 / 2}\right)$. Verifying this will complete the proof since the outer sum equals 1. By the Cauchy-Bunyakowsky-Schwarz Inequality, we see that

$$
\begin{aligned}
\sum_{k=0}^{\infty} b_{n, k} \frac{\sqrt{n}}{\sqrt{k+1}} & =\sum_{k=0}^{\infty} \sqrt{b_{n, k}} \frac{\sqrt{n}}{\sqrt{k+1}} \sqrt{b_{n, k}} \\
& \leq\left(\sum_{k=0}^{\infty} b_{n, k} \frac{n}{k+1}\right)^{1 / 2}\left(\sum_{k=0}^{\infty} b_{n, k}\right)^{1 / 2} \\
& =\left(\sum_{k=0}^{\infty} b_{n, k} \frac{n}{k+1}\right)^{1 / 2} \\
& =\left(\sum_{k=0}^{\infty} b_{n, k+1}\right)^{1 / 2} \\
& \leq 1
\end{aligned}
$$

The next result concerns a variation of statistical convergence based on $B^{*}$. It is convenient to rewrite the definition of st-lim $x=L$ as

$$
\lim _{n \leq n:\left|x_{k}-L\right| \geq \epsilon} \frac{1}{n}=0
$$

for every $\epsilon>0$. The " $1 / n$ " in the sum is the $n, k$-th term of the Cesàro matrix $C$ for $k \leq n$. We now replace $1 / n$ with the $n, k$-th term of $B^{*}$ and define $\left(B^{*}\right)$ st-lim 
$x=L$ provided that

$$
\lim _{n:\left|x_{k}-L\right| \geq \epsilon} b_{n k}=0
$$

for every $\epsilon>0$. The following theorem is the $B^{*}$-analog of Theorem 3 of [5].

Theorem 3.1. If the sequence $x$ satisfies $\left(B^{*}\right)$ st-lim $x=L$ and $\Delta x_{k}=O\left(k^{-1 / 2}\right)$, then $\lim x=L$.

Proof. The argument is similar to Hardy's proof of Theorem 137 in [7]. We assume without loss of generality that $L=0$ and suppose to the contrary that $\lim x \neq 0$. Then there exists an $\epsilon>0$ such that $\lim \sup _{k}\left|x_{k}\right|>3 \epsilon$. Choose a subsequence $\left\{x_{n(q)}\right\}$ satisfying $\left|x_{n(q)}\right|>2 \epsilon$ for each $q$. Since $\left|\Delta x_{k}\right| \leq K / \sqrt{k}$ for some $K>0$, we claim that for $\gamma=\epsilon / K$ we have

$$
\left|x_{k}\right|>\epsilon, \quad \text { for each } q \text { and } n(q) \leq k \leq n(q)+\gamma \sqrt{n(q)} .
$$

Indeed, for an arbitrary $q$ and $k \in[n(q), n(q)+\gamma \sqrt{n(q)}]$, we note that

$$
\begin{aligned}
\left|x_{k}\right| & =\left|x_{n(q)}+\sum_{j=n(q)}^{k-1} \Delta x_{j+1}\right| \\
& \geq\left|x_{n(q)}\right|-\sum_{j=n(q)}^{k-1}\left|\Delta x_{j+1}\right| \\
& >2 \epsilon-\sum_{j=n(q)}^{k-1} \frac{K}{\sqrt{j}} \\
& \geq 2 \epsilon-\frac{K(k-n(q))}{\sqrt{n(q)}} \\
& \geq 2 \epsilon-\frac{K \gamma \sqrt{n(q)}}{\sqrt{n(q)}} \\
& =\epsilon .
\end{aligned}
$$

Now $\left(B^{*}\right)$ st-lim $x=0$ gives that

$$
\begin{aligned}
0 & =\limsup _{q} \sum_{k:\left|x_{k}\right|>\epsilon} b_{n(q), k} \\
& \geq \limsup _{q} \sum_{k \in[n(q), n(q)+\gamma \sqrt{n(q)}]} b_{n(q), k} \\
& >0 .
\end{aligned}
$$

The last inequality follows by parts (4) and (5) of Hardy's Theorem 137 of [7. This contradiction completes the proof.

We are now prepared to prove a statistical extension of Hardy and Littlewood's Tauberian theorem [8] for Borel summability. In addition to Lemma 3.1 and Theorem 3.1, we shall also need the following result due to Bingham [2].

Lemma 3.2. If the sequence $x$ satisfies $\lim B^{*} x=L$ and $\Delta x_{k}=O(1 / \sqrt{k})$, then $\lim x=L$. 
The preceding lemma, which is obviously the matrix extension of Hardy and Littlewood's theorem, is not stated in this form by Bingham. This statement, however, follows immediately from his remark at the top of page 222 of [2] and Theorem $3 \mathrm{~b}$ on page 224 .

Theorem 3.2. If the sequence $x$ satisfies $\left(B^{*}\right)$ st-lim $B^{*} x=L$ and $\Delta x_{k}=O(1 / \sqrt{k})$, then $\lim x=L$.

Proof. By Lemma 3.1 the order hypothesis implies that $\left(\Delta B^{*} x\right)_{n}=O\left(n^{-1 / 2}\right)$, which allows us to apply Theorem 3.1 to $B^{*} x$ to infer that $\lim B^{*} x=L$. Now Lemma 3.2 can be invoked to conclude that $\lim x=L$.

The authors would like to thank the referee for several suggestions that have improved the exposition of this work.

\section{REFERENCES}

1. D. H. Armitage and I. J. Maddox, Discrete Abel mean, Analysis 10 (1990), 177-186. MR 91h:40006

2. N. H. Bingham, Tauberian theorems and the Central Limit Theorem, The Annals of Probability 9 (1981), no. 2, 221-231. MR 82f:40010

3. H. Fast, Sur la convergence statistique, Colloq. Math. 2 (1951), 241-244. MR 14:29

4. J. A. Fridy and M. K. Khan, Tauberian theorems via statistical convergence, J. Math. Anal. Appl. 228 (1998), no. 1, 73-95. CMP 99:05

5. J. A. Fridy, On statistical convergence, Analysis 5 (1985), 301-313. MR 87b:40001

6. G. H. Hardy, Theorems relating to the summability and convergence of slowly oscillating series, Proc. London Math. Soc. 8 (1910), no. 2, 310-320.

7. — Divergent series, 2nd edition, Oxford University Press, Oxford, 1949. MR 11:25a

8. G. H. Hardy and J. E. Littlewood, Theorems concerning the summability of series by Borel's exponential method, Rend. Circ. Mat. Palermo 41 (1910), no. 2, 36-53.

9. are positive, P. Lond. Math. Soc. 13 (1914), 174-191.

10. K. Knopp, Über das Eulershe Summierungsverfahren, Math. Zeit. 18 (1923), no. II, 125-156.

11. E. Landau, Über die Bedentung einiger Grenzwertsätze der Herren Hardy und Axer, Prace Mat.-fiz. 21 (1910), 97-177.

12. J. E. Littlewood, The converse of Abel's theorem on power series, P. Lond. Math. Soc. 9 (1910), no. 2, 434-448.

13. R. E. Powell and S. M. Shah, Summability theory and applications, Van Nostrand Reinhold, London, 1972.

14. R. Schmidt, Über divergente Folgen und Mittelbildungen, M. Zeit. 22 (1925), 89-152.

Department of Mathematics and Computer Science, Kent State University, Kent, Оніо 44242

E-mail address: fridy@mcs.kent.edu

E-mail address: kazim@mcs.kent.edu 